Les barres omnibus des feeders distribuent le courant sur 7 panneaux de feeders placés de part et d'autre des 3 tableaux de transformateurs. Les panneaux sont tous identiques. Ils comprennent chacun un disjoncteur automatique à rupture brusque dans l'huile de $\mathrm{I}$ ooo kilovoltampères et un ampèremètre par phase.

Le courant venant des barres passe par les transformateurs d'intensité du disjoncteur ( 2 sur 3 phases) et l'interrupteur; de là il traverse les transformateurs d'intensité des ampèremetres et va enfin rejoindre les boîtes d'extrémité des câbles-feeders.

Chaque pannealy ainsi constitué est encore relié à un autre tableau comprenant une série de 7 panneaux de limiteurs de tension. Le fonctionnement et le montage du tableau à 5000 volts sont du reste indiqués sur le schéma de montage de la sous-station.

Les disjoncteurs automatiques dont on a parlé ci-dessus ont aussi été construits par la Société Industrielle des Téléphones; ils sont réglabes et à action différée. Ainsi que nous lavons indiqué plus haut le mécanisme, de déclanchement est commandé par 2 phases seulement; dans ce systeme, il n'existe pas de relai; le courant secondaire des transformateurs $T$ montés en série passe directement dans

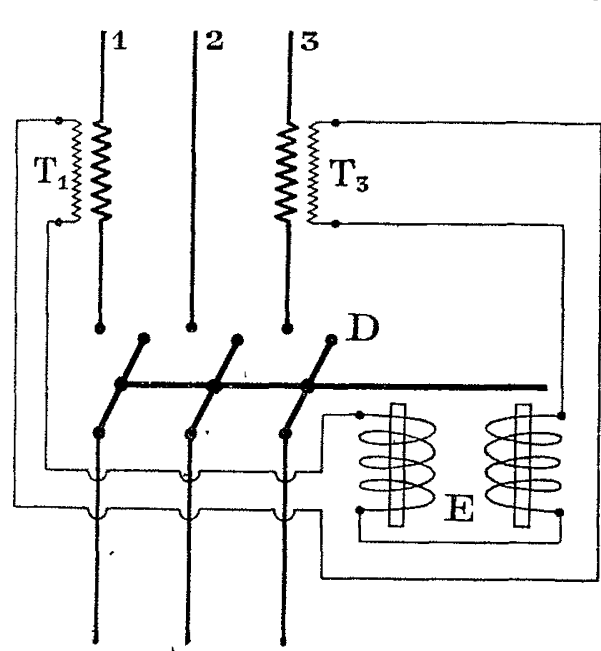

FIG. 6. - Interrupteurs S. I. T. 2 électro $E$ en série, dont le novau de fer doux mobile vient buter contre un levier de déclanchement, lorsque l'intensité atteint une trop grande valeur. Le réglage de cet appareil s'obtient en faisant varier la course du noyau mobile,et par conséquent la distance d'attraction; le retard, lui, est obtenu en faisant varier la course d'un piston dans un cylindre à glycérine, relié au mécanisme de déclanchement. Le montage électrique de cet appareil est du reste représenté sur le schéma ci-contre (fig. 6).

Les ampèremètres, de provenance Hartmann et Braun, de Francfort, sont à transformateurs d'intensité.

Chaque phase traverse un transformateur d'ampèremètre et les secondaires de ces appareils sont réunis a un commutateur bipolaire à 3 directions qui permet de mesurer l'intensité sur chaque fil au moyen d'un seul cadran.

Le tableau proprement dit comprend encore un voltmètre ordinaire, et un voltmètre enregistreur thermique, système Jules Richard. Ces deux appareils sont branchés sur les secondaires de transformateurs triphasés 5000 volts/ 1 o volts, par l'intermédiaire de commutateurs bipolaires à 3 directions permettant de mesurer le voltage sur les 3 ponts. Ainsi qu'on a pu le remarquer, aucun des fils à haute tension ne communique directement avec la partie accessible du tableau.

Il nous reste enfin à décrire les panneaux de limiteurs de tensions. Ces appareils sont du système Vedovelli; ils se composent d'une série de huit obus en zinc strié, séparés les uns des autres par un intervalle d'air de un millimètre environ. Ils sont réglés pour s'amorcer à un voltage de $\frac{7500}{\sqrt{3}}$ volts par rapport à la terre, soit pour une surtension de 50 pour roo de voltage normal. Ils sont reliés aux bornes de départ des feeders par l'intermédiaire du coupecırcuit à huile CC destiné à couper le courant de décharge au cas où celui-ci atteindrait une trop grande valeur.

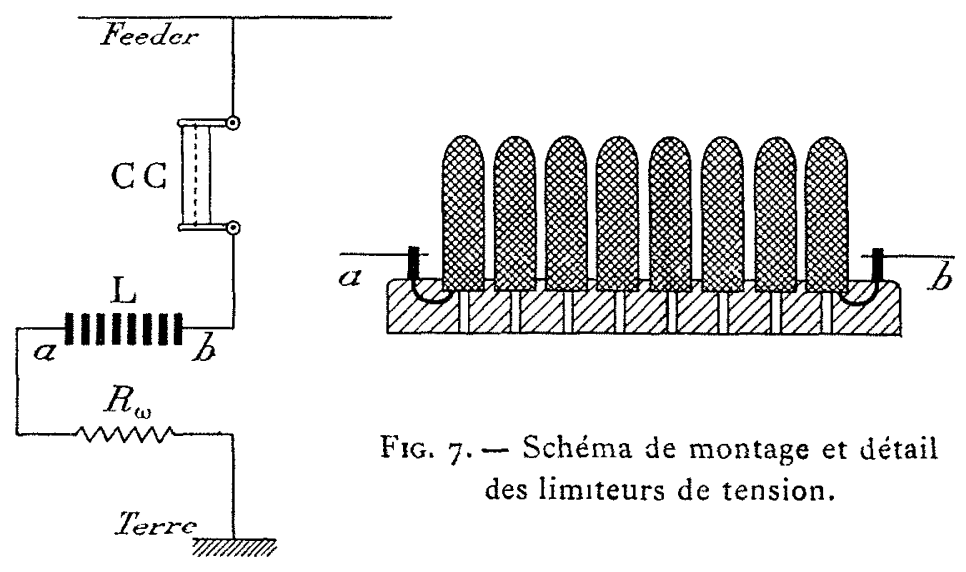

Ces coupe-circuits à huile, d'un modèle très ingénieux, et qui donne les meilleurs résultats, sont construits par la maison Sprecher et Schuler, d'Aarau (Suisse); ils sont amovibles et peuvent se manœuvrer à vide au moyen de pinces isolantes spécialement combinées à cet effet. Les fusibles, en argent, sont maintenus tendus au moyen d'un tube de porcelaine rempli d'huile. Lorsque le fusible vient à se rompre, à la suite d'une surintensité, le ressort attire vivement l'arc dans l'huile et celui-ci est alors étouffé instan. tanément.

Enfin, sur le circuit de terre de ces limiteurs, se trouvent branchées en série des résistances ohmiques qui sont destinées à limiter le courant de décharge.
(A suivre.)
F. JENNY.

\section{Redresseur-Régulateur Auvert et Ferrand}

Dans le dernier numéro de La Houille Blanche, on a lu le rapport présenté par M. Auvert au 35e Congrès de l'Associalion française pour l'avancement des Sciences sur la tracticn électrique des chemins de fer; nous donnons aujourd'hul la description du redresseur-régulateur de courant alternatif en courant continu de M. Auvert, d'après un article qu'il a publié sur cet appareil dans la Revue Générale des chemins de fer el Tramways.

Le but de tout redresseur est de transformer un courant alternatif monophasé en un courant qui soit, sinon tout à fait continu, du moins toujours de même sens. L'appareil imaginé par MM. Auvert,ingénieur principal, et Ferrand, ingénieur à la Cie P.-L.-M., permet, grâce à un artifice spécial, d'obtenir un courant qui, non seulement est toujours de même sens, mais encore est très peu ondulé. Il repose sur le principe suivant :

Considérons un anneau lamellé AA (fig. 1) formé de minces lames de tôle d'acier doux, autour duquel sont placés deux enroulements EE occupant chacun le quart de la circonférence de l'anneau. Sur le même axe que celui ci est monté un collecteur ayant l'aspect extérieurd'un collecteur de dynamo à courant continu, mais quí en diffère cependant en ce qu'il est divisé en quatre parties égales, dont deux XY sont formées de lames isolées les unes dos autres par du mica, et dont les deux autres $B$ et $C$ sont formées d'une portion de cylindre remplissant l'intervalle compris entre les parties lamellées. 
Les lames isolées sont respectivement reliées aux différentes sections des enroulements comme dans un anneau Gramme, et les extrémités de ces enroulements sont reliées en $f g h k$ aux parties B et C non sectionnées du collecteur. La partie non sectionnée $B$ est reliée à une bague $M$ et la partie non sectionnée $\mathrm{C}$ est reliée à une bague $\mathrm{L}$.

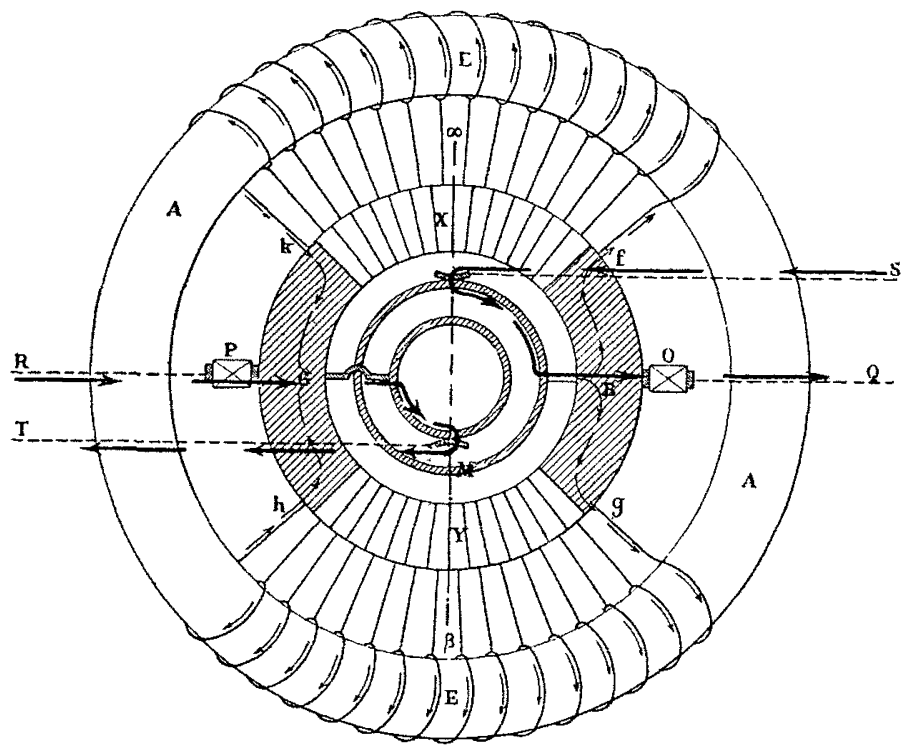

FıG. 1. - Premier schéma du redresseur-rígulateur.

Les bagues $L$ et $M$ sont en communication, au moyen de frotteurs, avec les pôles $T$ et $S$ d'une source de courant alternatif monophasé, et deux balais $\mathrm{O}$ et $\mathrm{P}$, qui appuient sur le collecteur, en deux points opposés, servent à recueillir le courant redressé.

Supposons tout d'abord que l'appareil reste immobile; il est aisé de voir, en examiuant le sens des enroulements, qu'un courant alternatif arrivant des points $\mathrm{S}$ et $\mathrm{T}$ par les frotteurs et les bagues, parcourra les enroulements EE de telle sorte que les flux magnétiques engendrés dans les deux parties de l'anneau seront concordants. Le flux tótal parcourant l'anneau sera fermé sur lui-même comme dans le noyau d'un transformateur. On obtiendra, aux points $Q$

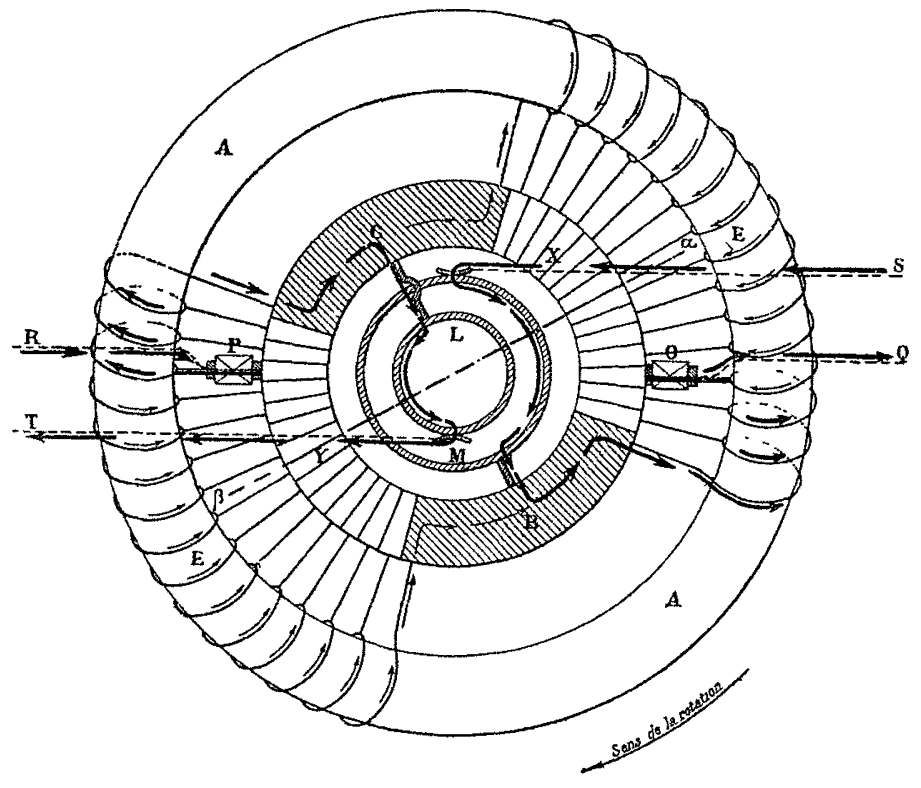

Fıa. 2. - Deuxième schéma du redresseur-régulateur

et $R$, des tensions alternatives de mème périodicité et de même phase qu'aux points $\mathrm{S}$ et $\mathrm{T}$, et si les points $\mathrm{Q}$ et $\mathrm{T}$ sont reliés à un appareil d'utilisation, un moteur par exemple, il est clair que tout se passera comme si ce moteur était directement alimenté par la source du courant alternatif. (Le tracé en trait plein indique la marche du courant princi- pal). Quant aux enroulements EE, ils sont parcourus par un faible courant secondaire, juste celui nécessaire pour créer, par réaction de self-induction, entre les points $f$ et $k, g$ et $h$, une force électro-motrice égale et opposée à la tension al. ternative de la source.

Admettons maintenant que l'on fasse tourner l'anneau à la vitesse du synchronisme, et que, de plus, le mouvement ait été réglé de telle sorte que les balais $\mathrm{O}$ et $\mathrm{P}$ soient au milieu des parties non sectionnées $B$ et $C$ au mument oú la tension alternative est maximum. La moitié $\alpha \mathrm{B} \beta$ du collecteur a toujours la même polarité que le point $S$, tandis que la deuxième moitiá $\alpha \mathrm{C} \beta$ a toujours la même polarité que le point $\mathrm{T}$.

L'anneau tournant à la vitesse du synchronisme, chacune des moitiés du collecteur passera devant chacun des frotteurs $O$ et $P$, précisément dans le temps qu'elle aura une polarité déterminée, et celle-ci changera de signe au momen même où chaque moitié du collecteur quittera un frotteur pour venir en contact avec l'autre frotteur. En d'autres termes, la tension aux frotteurs $\mathrm{O}$ et $\mathrm{P}$-sera toujours de même sens, et l'appareil fournira du courant redressé.

Tant que les frotteurs $O$ et $P$ seront en contact avec les parties pleines B et $\mathrm{C}$ du collecteur, les points $Q$ et $R$ seront en communication directe avec la source alternative, et les enroulemients EE ne seront parcourus que par un courant dérivé très faible. Au contraire, quand les frotteurs seront en contact avec les parties sectionnées du collecteur, les points $Q$ et, $R$ seront en communication indirecte avec la source alternative par l'intermédiaire des enroulements $\mathrm{EE}$. Les intensités des courants qui circulent dans les deux parties de chaque enroulement, placées de part et d'autre de chaque balai, sont constamment variables avec la position angulaire de l'anneau, mais la somme algébrique de leurs ampères-tours est toujours à chaque instant égale $\dot{a}$ celle qui, dans la marche à vide, est nécessaire pour créer une force électro-motrice de self-inductirın égale et contraire à celle de la source.

La démonstration précédente suppose que les deux halais $\mathrm{O}$ et $\mathrm{P}$ sont calés à $180^{\circ}$ l'un de l'autre. Dans ces conditions, si on alimente l'appareil aux points $\mathrm{S}$ et $\mathrm{T}$ avec une source de courant alternatif ayant un voltage efficace constant, on obtient, aux balais $\mathrm{O}$ et $\mathrm{P}$, du courant redressé ayant un voltage constant, quelque soit le débit. Il ness'en suit pas que la tension entre $\mathrm{O}$ et $\mathrm{P}$ soit constante pendant la durée d'une période, mais seulement que la moyenne arilhmétique des tensions, pendant la durée d'une période, est cons. tante et toujours de même sens $\left(^{\star}\right)$.

Si au lieu de maintenir les deux balais $\mathrm{O}$ et $\mathrm{P}$ calés à $180^{\circ}$ l'un de l'autre, on rapproche progressivement l'un d'eux, O par exemple, de l'autre balai $P$ supposé fixe, on constate, par les indications du voltmètre, que la tension entre les deux balais $\mathrm{O}$ et $\mathrm{P}$ va en décroissant au fur et $\dot{a}$ mesure que l'angle qui les sépare devient plus petit. Celte tension se réduit à zéro lor'sque les deux balais viennent en coïncidence. Cet appareil redresseur peut donc nun selllement servir à transformer du courant alternatif monophasé en courant redressé, mais aussi à obtenir du courant redressé à tension variable à volonté. C'est là une propriété précieuse, qui permet d'augmenter considérablement le champ d'utilisation de cet appareil.

Dans l'appareil schématique ainsi décrit, l'anneau n'est muni que d'un collecteur, auquel correspondent deux enroulements occupant chacun le quart de la circonlérence, de sorte que la moitié de l'anneau est complètement inutilisée. Pour y remédier, il suffit de munir l'appareil d'un

(*) C'est-à-dire qu'un voltmètre à aimant permanent, branché entre les deux balais 0 et $P$ donnerait une indication constante. La tension vapie en réalité, pendant une période, depuis zéro jusıu'à un maximum, toul en restant au-dessus de zéro, mais ses variations sont trop rapides pour en restant au-dessus de zéro, mals ses variations sont trop rapent la
ètre survies par l'aiguille du voltmètre. lequel prend exactement sition correspondant à la moyenne arithmétıque des deux tensions. 
deuxième collecteur, symétrique du premier par rapport à l'anneau, et de disposer sur cet anneau deux autres enroulements occupant l'espace disponible. Les nouvelles connexions étant identiques à celles déjà décrites, on a airısi, sur le même arbre, deux redresseurs qui peuvent être confondus en un seul en reliant en parallèle, par des connexions convenables, les balais fixes et mobiles de même polarité, ainsi que les parties similaires non sectionnées des collecteurs.

Jusqu'ici, nous n'avons considéré que le cas d'un redresseur bipolaire; or, pour une fréquence de 25 périodes seulement, qui est tout à fait usuelle, un redresseur bipolaire doit faire 1500 tours par minute. D'autre part, lorsqu'on veut recueillir des courants de grande intensité, on est obligé, pour ne pas trop allonger les collecteurs, d'employer plusieur's lignes de balais en construisant des redresseurs multipolaires. Ceux ci, qui ont l'avantage de tourner à une vitesse relativement faible, dérivent du redresseur bipolaire exactement comme s'il s'agissait de dynamo à courant continu. teurs distincts pour alimenter le régulateur-redresseur, mais les circuits primaires étaient montés en parallèle.

Pour essayer le redresseur-régulateur dans les conditions se rapprochant le plus possible de colles qu'on rencontrerait dans une application à une locomotive, on envoya le courant redressé dans quatre moteurs série hexapolaires. Pour freiner ces moteurs, on leur faisait conduire à chacun une dynamo génératrice, à excitation séparée, et le courant produit était absorbé dans des rhéostats.

Sur l'un des câbles reliant le redresseur-régulateur à l'ensemble des quatre motcurs. on avait intercalé une bobine de self-induction, constiluée par le secondaire d'un transformateur statique dont le primaire réstait ouvert, ceci dans le but de supprimer on grande partie les ondulations du courant et de la teusion aux bornes des moteurs $\left({ }^{*}\right)$.

Des appareils de mesures permettaient de se rondre comple des quantités d'énergie fournies au redresseur-régulateur et restituées par lui. Enfin, un ondographe Hospitalicr permettait de relever graphiquement les variations, pendant une période, de la tension ct de l'intensité du courant alternatif monophasé, ainsi que du courant redressé obtenu pour différentes positions des balais (fig. 4, 5 et 6 ).

Pour une tension alternative de 165 à 167 voltss, la tension moyenne totale du courant redressé, mesurée au voltmètre magnétique, variait de 22 à 280 volts, suivant la position des balais mobiles.

On a trouvé que le rendement électrique (non compris la puissance fournie au moteur synchrone qui entretient le mouvement de l'appareil) était de 91 pour 100 à la puissance de $400 \mathrm{kw}$, lorsque les balais mobiles étaient au maximum de leur course ; qu'il restait égal à 91 pour' 100 , pour $200 \mathrm{kw}$, lorsque les balais sont à mi-course ; et qu'il était encore de 87,5 pour 100 , pour $105 \mathrm{kw}$, lorsque les halais mobiles étaient au quart de leur

Fig. 3. - Schéma des comexions d'un groupe de deux redresseurs.
Un redresseur-régulateur d'essai fut construit sur ces principes aux ateliers de Champagne-sur-Seine de la Société Schneider et $\mathrm{C}^{1 \mathrm{e}}$, et essayé à la station centrale de la Compagnie du Métropolitain de Paris, à Bercy. Ce redresseur-régulateur était tétrapolaire, et se composait de deux redresseur's montés en série du côté continu, et complètement indépendants du côté alternatif. Chacun des redresseurs était tétrapolaire avec collecteurs en parallc̀le, et ils étaient entraînés tous deux par un même moteur synchrone lournant à la vitesse de 750 tour's par minute, sous une fréquence de 25 périodes par seconde.

Les deux bagues de chaque redresseur sont en communi. cation, au moyen de frotleurs $f f$ (voir firg. 3), avec les pôles d'une source de courant alternatif monophasé, laquelle était constituée par le secondaire d'un transformateur statique fournissant du courant a la tension de 160 volts environ, et dont le primaire était alimenté par du courant alternatif monophasé à 5000 volts, pris sur l un des circuits du réseau triphasé du Métropolitain. Il y avait ainsi deux transforma- course. C'est-à-dire que le rendement électrique varie fort peu lorsque l'on réduit le voltage. Quant au rendement réel du régulateur-redresseur, il ressort à 88,4 pour 100 pour $400 \mathrm{kw}$; à 86,2 pour 100 pour $200 \mathrm{kw}$ et à 79,5 pour 100 pour $105 \mathrm{kiv}$.

Ce sont là de bons rendements, surtout pour un apparcil d'essais. Ils seraient certainement dépassés en cas de construction de nouveaux redresseurs-régulateurs de plus grande puissance.

Au point de vue de la commutation, le fonctionnement de l'appareil est bon et il ne se produit pas d'étincelles nuisibles sur les collecteurs. De même la stabilití est excellente. C'est ainsi que, l'appareil étant en pleine charge, on a pu dix fois de suite couper, puis rétablir le courant alternatif

(*) Parce que les moteurs de freinage avaient des inducteurs massifs en acier coulé dont la self-induction étail naturellement faible. Dans le cas où lon emplonerait des inducteurs feuilletés, Ja réaction de selfinduction deviendrail suffisante, et l'on pourrait supprimer la bohine de self. 
qui alimentait le moteur synchrone sans faire décrocher celui-ci. On a terminé l'essai en supprimant le courant alternatif qui alimente le moteur synchrone, tout en laissant en pleine charge le redresseur-régulateur. Celui-ci se met hors phase et s'arrête progressivement. Le

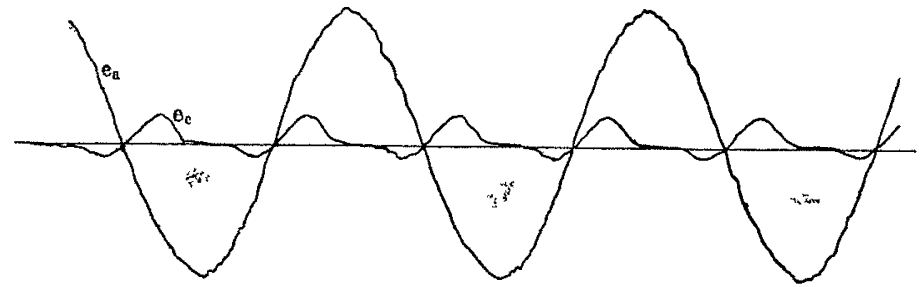

Fig. 4. - Les balais mobrles sont au commencement de la course, dans la position la plus rapprocliée des balas tixes. $e$ a tension alternatire, lof volts elficaces,

$e_{\mathrm{c}}$ teusion totale moyenne redressée, 22 volts.

courant redressé est remplacé par du courant allernatif dont l'intensité est forcément très réduite par la forte selfinduction du circuit. D'allleurs il ne se produit rien d'anormal sur les collecteurs qui continuent à fonclionner sans étincelles.

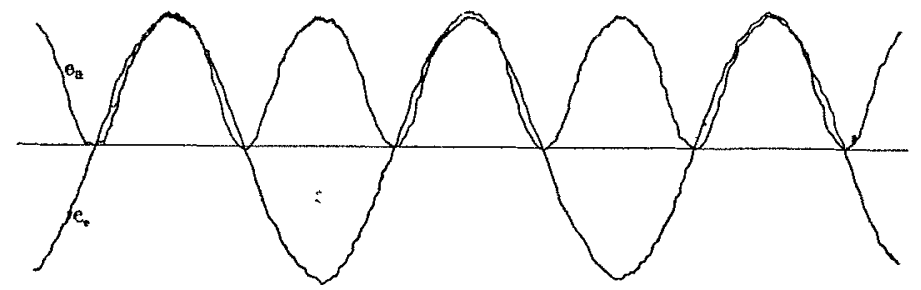

Fig. 5. - Les balass molıles sonl à fond de course.

ea tension alternative, 167 rolts efficaces, ec tension totale moyenne 230 rols.

Un avantage particulier du redresseur-régulateur réside dans ce fait qu'il se prête avec la plus grande facilité à la récupération de l'énergie, car' cet appareil est entièrement réversible. Et si l'on emploie sur les locomotives des moteurs à excitation séparée, on pourra maintenir sur les déclivités telle vitesse que l'on voudra, sans faire usage des freins, et en restituant à la ligne l'énergie qui serait inutilement absorbée par les sabots des freins. Enfin, il serait possible de faire usage de batteries tampons, comme on le fait pour la traction par courant continu (*).

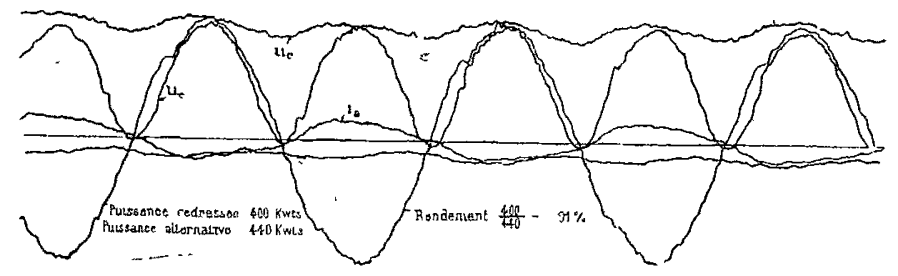

Fig 6. - Les balais mobiles sont à fond de course dans la posilion la plus éloıgnée des balais fixes.

$U_{\mathrm{a}}=155$ volts efficaces $U_{\mathrm{c}}=235$ volts moyen.

$i_{\mathrm{a}}=1390 \mathrm{amp}$. efficaces $i_{\mathrm{c}}=1500 \mathrm{amp}$. moyen.

Un calcul détaillé, fait en vue de l'application de la traction électrique sur la ligne d'Alais à Langogne, a montré qu'en employant le régulateur-redresseur on pourrait, grâce à la récupération, diminuer de 27 pour 100 la quantité d'énergie électrique. En tout cas, on conçoit sans peine qu'on réaliserait une économie en employant cet appareil sur les lignes à profil très accidenté $\left.{ }^{* *}\right)$.

(*) Ces batteries seraient, bien entendu, placées dans la station centrale, ou dans des sous-stations appropriées.

(“) Lors de sa communication au Congrès de Lyon, M. Auvert a déclaré que, à la suite d'un concours, son redresseur-régulateur avait été adopté pour l'alımentation des locomotives qui feront le service sous le tunnel du Loechesberg dont le percement vient d'ètre décidé.

\section{LE MOIS HYDRO-ÉLECTRIQUE}

\section{ACADÉMIE DES SCIENCES}

\section{MÉCANIQUE ET ÉLECTRICITÉ}

Résistance des électrolytes pour les courants de haute fréquence. - Note de MM. André Broca et S. Turchini, séance du 28 mai r 906 .

La théorie de Lord Kelvin relatıve à la résistance des conducteurs cylindriques pour les courants de haute fréquence condurt, dans le cas des métaux, à des résultats qui présentent avec l'expérience des différences systématiques, comme nous l'avons montré ll y a un an (Comples rendus, t. CXL, p. 1228). Nous avons repris ces expériences en nous adressant aux électrolytes, pour lesquels la théorie doit s'appliquer comme pour les métaux, espérant trouver des divergences analogues. La difficulté était seulement de rédiser un conducteur électrolytıque assez gros pour que les effets de concentration à la surface pussent s'y produire, malgré la faible conductıbilité de ces corps. Nous avons opéré sur un cylındre de $6 \mathrm{~cm}$ de diamètre et de $10 \mathrm{~cm}$ de long, et nous avons eu des résultats nets en employant l'eau acidulée, à partir d'une concentration suffisante.

Nous avons commencé par vérifier, au moyen de notre électrodynamomètre précédemment décrit, qu'un ampèremètre à fil chaud spécralement construit pour les courants de haute fréquence donnait des indications exactes dans les limites de période entre lesquelles nous avons opéré ; cela a rendu les détermınations ultérieures beaucoup plus faciles. Les perturbations dues a la tréquence deviennent négligeables dans le cas de fils aussı fins que ceux quı sont employés dans ces appareils.

L.e princıpe de la méthode est le même que dans notre étude des fils métalliques. On mesure d'une part l'intensité efficace du courant de haute fréquence, et d'autre part l'échauffement qu'il produst dans le conducteur électrolytique, en le traversant pendant une minute. On recommence l'éxpérience en mesurant l'échauffement produit dans ce même conducteur par un courant alternatif à 42 périodes passant pendant le mème temps et ayant la même intensité efficace. Le rapport des deux échauffements donne le rapport des résistances du conducteur pour le courant de haute fréquence et pour le courant alternatıf ordinaire. Nous admettons que, pour la très basse fréquence de celui-ci, la résistance est la même qu'en courant continu, et nous pouvons, par son emploi, nous mettre à l'abri des phéno. mènes délectrolyse.

Pour mesurer l'echauffement dû au passage du courant, le tube qui contient le liquide est munı d'un tube capillaire latéral, dans lequel on mesure lascension du liquide échauffé. Le courant est amené dans l'appareil par deux larges électrodes de platune de $6 \mathrm{~cm}$ de dramètre. 'Tout l'appareil est soigneusement enveloppé de coton pour le mettre à l'abri des courants d'air. On commence toujours par lire pendant quelques minutes la marche normale de réchauffement ou de refroldissement de l'appareil, et on opère quand cette marche est bien constante et ne dépasse pas le vingtrème de l'échauffement attendu.

On peut voir que, dans les circonstances où nous sommes, les effets observés sont dus uniquement à l'échauffement, et que les modifications chimiques n'y entrent pour rien. Nous avons en effet des électrodes absolument symétriques, et toutes les réactions réversibles par nature sont éliminées par le tait même. S'll s'en passe qui ne le soient pas, elles ne peuvent non plus avorr d'action sensible. $\mathrm{M}$. Rothé a montré en effet que les électrodes prenaient, au bout d'un très petit nombre de cycles de potentiel, un état permanent qui correspond à un cycle de polarisation toujours identique à luimêne. D'allleurs, quand la dilution devient assez grande, le fast que les deux espèces de courant donnent la même chose, semble bren prouver qu'il en est ainsi. De plus, dans le cas du sulfate de cuivre, les résultats sont les mêmes avec des électrodes en cuivre et avec des electrodes en platine.

Nous avons ainsi obtenu le résultat suivant, en appelant $R_{f}$ la résistance en haute fréquence et $R_{c}$ la résistance à basse fréquence, supposée égale à celle qui existe en courant contınu.

Eau acidulée très étendue de sulfate de cuivre $\frac{R_{f}}{R_{c}}=\mathrm{I}$

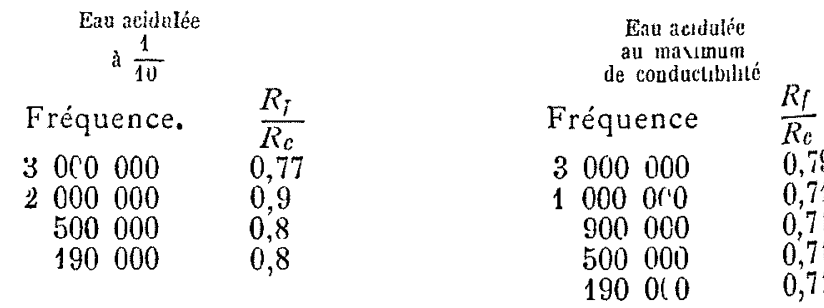

En somme, nous arrivons à ce résultat surprenant : 